\title{
EFECTO DE UN PROGRAMA DE INTERVENCIÓN EDUCATIVA SOBRE EL CONOCIMIENTO DE SEGURIDAD DE PACIENTES EN ESTUDIANTES DE PREGRADO DE ENFERMERÍA
}

\author{
EFFECT OF AN EDUCATIONAL INTERVENTION PROGRAM \\ ON PATIENT SAFETY KNOWLEDGE AMONG UNDERGRADUATE \\ NURSING STUDENTS
}

\author{
Bernardita Moreno Lobos* \\ Naldy Febré Vergara **
}

\begin{abstract}
RESUMEN
Objetivo: El objetivo de la presente investigación fue evaluar el efecto de un programa de intervención educativo sobre el conocimiento de seguridad de pacientes en estudiantes de Enfermería que se encuentran en su práctica clínica. Material y método: Estudio pre-experimental antes-después de 6 meses de duración, realizado en tres etapas. Muestra de 80 estudiantes de segundo año de la carrera de Enfermería, de una universidad privada. Recolección de datos, mediante aplicación de un instrumento tipo encuesta validado, con cinco secciones, el cual fue aplicado en dos periodos (antes y después de la intervención). La intervención consistió en la aplicación de metodología educativa con el apoyo de video instruccional de una hora de duración. Fueron respetados los requisitos éticos de investigación científica. Se efectuó análisis estadístico mediante la utilización de medidas de tendencia central y se utilizó estadística no paramétrica y paramétrica. Resultados: Se verificó un aumento en el porcentaje de logro de los conocimientos de los educandos, esto en relación a la pre (30,5\%) y pos (52,25\%) intervención educativa ( $\mathrm{p}$ valor $<0,05$ ). Conclusión: La intervención realizada logro aumentar el conocimiento por parte de los estudiantes en temas básicos de seguridad clínica.
\end{abstract}

Palabras clave: Seguridad del paciente, educación en enfermería, estudiantes de Enfermería.

\begin{abstract}
Objective: The main objective of this investigation was to evaluate the impact of an educational intervention program on patient safety knowledge among Nursing students who are in their clinical internship. Materials and methods: Before-after pre-experimental study, 6 months length, developed in 3 stages. Sample of 80 second year Nursing students from a private university. Data recollection was carried out through a validated survey-type instrument, consisting of five sections, which was applied in two periods (before and after the intervention). The intervention consisted in the application of educational methodology with the support of an hour-length video. The ethical principles required were respected. Statistical analysis through measures of central tendency, and parametric as well as nonparametric statistics were used. Results: The data showed an increase in learners knowledge, based on before $(30.5 \%)$ and after $(52.25 \%)$ educational intervention ( $\mathrm{p}$ value $<0.05)$. Conclusion: The intervention performed was able to increase students' knowledge in basic clinical safety issues.
\end{abstract}

Key words: Patient safety, nursing education, Nursing students.

Fecha recepción: 22/03/14 Fecha aceptación: 13/06/16

\footnotetext{
* Enfermera, Magíster en gestión de seguridad de pacientes y calidad de atención. Clínica Alemana. E-mail: berni.morenolobos@gmail.com

** Doctora en Ciencias de la Salud. Universidad Andrés Bello, Facultad de Enfermería. Autor de referencia, E-mail: naldy. febre@unab.cl
} 


\section{INTRODUCCIÓN}

La seguridad en la atención de salud representa uno de los temas prioritarios tanto a nivel mundial como nacional. Por este motivo, desde el año 2004 los estados miembros de la Organización Mundial de la Salud (OMS) formaron la "Alianza Mundial para la Seguridad del paciente", la cual tiene como fin principal impulsar la investigación como elemento esencial, para mejorar la seguridad en la atención sanitaria (1). En Chile, la primera manifestación de interés en el tema de la seguridad del paciente fue con la Reforma de Salud, Ley $\mathrm{N}^{\circ}$ 19.937, que establece una nueva concepción de la autoridad sanitaria (2). En el año 2005, la Superintendencia de Salud explicita que desde el punto de vista de la política pública, el Estado definió la calidad en la atención de salud como "aquel nivel en el cual los establecimientos y los profesionales otorgan sus prestaciones garantizando la seguridad de sus pacientes" (3).

A nivel mundial, en las últimas décadas los eventos adversos (EA) que generaron mayor interés investigativo fueron las infecciones asociadas a la atención de salud (IAAS) (4). Actualmente, se reconoce que otros eventos adversos incrementan significativamente los costos y la morbimortalidad de los pacientes hospitalizados, en especial, úlceras por presión, caídas de pacientes, errores de medicación, cirugía en paciente o sitio equivocado, identificación incorrecta de pacientes, entre otros; situación que ha producido cambios a nivel político, económico y social, mediante la construcción de nuevas instituciones normativas de carácter sanitario (4).

Debido a la importancia indiscutida de la seguridad del paciente, como dimensión clave de la calidad asistencial y su impacto en las políticas sanitarias a nivel mundial, se hace relevante que los profesionales enfermeros trabajen en perfeccionar la calidad de la formación, la asistencia, la investigación y la gestión del cuidado, para lograr dos obje- tivos comunes: 1) Cultura de seguridad y 2) Seguridad del paciente (5).

La seguridad clínica debe ser objetivo clave de la educación de Enfermería en pregrado, donde es fundamental instruir a los futuros profesionales en temas relevantes como la calidad del cuidado, epidemiología de los eventos adversos, además de su prevención y control $(6,7)$.

Una revisión integrativa realizada el año 2014 mostró que el contenido de seguridad del paciente no se incorpora formalmente en los programas de asignaturas (7), evidenciando la inexistencia de cambios en la tradicional formación de los futuros profesionales (8).

En el Manifiesto de Seguridad, publicado en 2011, los autores llaman a los educadores a una revisión crítica de los planes de estudio para incluir en el proceso de enseñanza-aprendizaje actividades centradas en la seguridad del paciente, con el fin de generar cambios curriculares; con énfasis en la integración de estrategias, que desarrollen las habilidades de los estudiantes para el razonamiento y juicio clínico (9).

La definición de seguridad del paciente, entregada por la Organización Mundial de la Salud (OMS), es "la ausencia, para un paciente, de daño innecesario o daño potencial asociado a la atención sanitaria” (10). En el año 2007 esta organización publica "Nueve soluciones para la seguridad del paciente", basada en la implementación de sistemas de vigilancia para disminuir la probabilidad de errores y maximizar la posibilidad de interceptarlos cuando ocurran (11).

Los estudiantes de Enfermería, que se enfrentan a prácticas clínicas en contacto con pacientes, deben tener conocimiento sobre la seguridad de pacientes, ya que esto favorece la calidad de la atención brindada y previene los posibles eventos adversos que se puedan generar (4). Especial mención merecen los estudiantes que inician por primera vez sus prácticas clínicas, los cuales no han tenido la oportunidad de desarrollar una conciencia 
de riesgo para el paciente, problemas de seguridad, o un sentido claro de la responsabilidad que recae en el personal de enfermería $(6,8)$. En ese entendido, la estructuración del plan de enfermería supone tener en cuenta el riesgo específico de cada paciente, así como el contexto organizacional en el cual se actúa profesionalmente (12).

Considerando lo anteriormente expuesto, es indispensable que esta temática sea incluida en todos los planes de estudio de la carrera de Enfermería, con la finalidad que los alumnos conozcan los factores que intervienen en la ocurrencia de estos eventos adversos y así sepan cuáles son los recursos indispensables que se requieren para realizar los diferentes procedimientos para dar cuidado, de manera que puedan evitar errores en la atención (13).

En el año 2014 se realizó un estudio cuyo objetivo fue evaluar los errores de medicación producidos por estudiantes de Enfermería, donde los autores concluyen que los estudiantes en formación deben recibir información sobre seguridad clínica y errores de medicación al inicio del proceso educativo, lo que permitirá mayor transparencia en la notificación de eventos adversos (errores de medicación) y aumento de la seguridad de los pacientes a su cargo (14).

La formación desde pregrado de esta importante área de desarrollo es indispensable, para aumentar el conocimiento en este tema, estructurando una cultura de seguridad en el colectivo enfermero, con el objetivo de colaboración entre las diferentes escuelas de Enfermería, para poder compartir y aprender de las iniciativas derivadas del estudio de los EA, las metas internacionales de seguridad del paciente, las nueve soluciones para la seguridad del paciente y así mejorar la seguridad de las personas bajo el cuidado enfermero (15, 16). Este desarrollo de nuevas competencias permitirá que la disciplina y profesión de enfermería integren y desarrollen estrategias que garanticen la calidad y seguridad clínica, tanto para los pacientes como para las profesiones sanitarias (11).
Por lo anteriormente expuesto, el objetivo de la presente investigación es evaluar el efecto de un programa de intervención educativa sobre el conocimiento de temas básicos de seguridad de pacientes (metas internacionales de seguridad y cultura de seguridad) en estudiantes de pregrado de una escuela de Enfermería privada que cursan su primera práctica clínica.

La hipótesis a comprobar plantea que los estudiantes sometidos a la intervención educativa tendrán un aumento de conocimientos significativo respecto a la seguridad de los pacientes que el evidenciado previo a la intervención.

\section{MATERIAL Y MÉTODO}

Para el logro del objetivo se diseñó un estudio preexperimental antes-después de 6 meses de duración, realizado en tres etapas.

Población objetivo: 95 estudiantes de pregrado de segundo año de la carrera de Enfermería de la institución en estudio, que cursaban su primera práctica clínica. Se calculó una muestra de 34 estudiantes, con un 95\% de confianza y un error alfa igual a $5 \%$. No obstante, 80 estudiantes se interesaron para formar parte del presente estudio (porcentaje de participación $84 \%$ del universo). Criterio de inclusión: todos los estudiantes de segundo año de la carrera de Enfermería de la institución en estudio, que se encontraban cursando su primera práctica clínica. Criterios de exclusión: estudiantes con trabajo previo en el área de la salud, alumnos repitentes, alumnos trasladados de otra universidad.

Para el desarrollo del estudio se aplicaron las tres etapas definidas:

a) Primera etapa: se invitó a los estudiantes a participar de la investigación, mediante información entregada por los autores en reunión, en la cual los estudiantes que aceptaron participar debieron firmar un consen- 
timiento informado. En este grupo se aplicó una encuesta desarrollada por los autores y validada previamente por 3 expertos de distintas instituciones, vinculadas al área calidad y seguridad del paciente, los que fueron consultados acerca de su opinión experta sobre el instrumento creado por los investigadores. Para esto se realizaron dos rondas de consulta, con el objetivo de comprobar que los diferentes ítems y dimensiones de la primera versión del instrumento permitieran medir los constructos o dominios originales, además de evaluar si el lenguaje empleado se adapta a la población en la que se aplicaría, es decir, la comprensión de las preguntas y la pertinencia de las opciones de respuesta. Las observaciones y los cambios propuestos por los integrantes del grupo de expertos fueron revisadas por los investigadores del estudio para posteriormente incluirlas y finalizar con una segunda versión consensuada del instrumento.

Para la validación en campo, la versión final se aplicó a un grupo de 20 estudiantes de Enfermería de tercer año, en formato impreso. Se observó el tiempo que los estudiantes ocupan en completar el instrumento, preguntas o datos que les generen duda o confusión y que no lograsen responder. Se evaluó la consistencia interna aplicando el coeficiente alfa de Cronbach (valor igual a 0,82).

La encuesta aplicada, pre y posintervención, presentaba cinco secciones: Sección A: datos sociodemográficos del estudiante; Sección B: evaluación de los conocimientos del estudiante respecto a seguridad de pacientes con preguntas de verdadero y falso; Sección C: evaluación de la percepción sobre el campo clínico en cuanto a cultura de seguridad; Sección D: valoración de la cultura de seguridad de los estudiantes; Sección E: valoración sobre las herramientas y contenidos entregados por la universidad respecto a seguridad de pacientes. Las secciones C, D y E se presentaban en escala tipo Likert frente a cada afirmación, siendo las respuestas clasificadas en totalmente desacuerdo; en desacuerdo, neutral, de acuerdo, totalmente de acuerdo. Cada sección, a excepción de la A, contaba con puntaje máximo de 7 puntos sección $B$, 13 puntos sección $\mathrm{C}$; 11 puntos sección $\mathrm{D}$ y 9 puntos sección E.

b) Segunda etapa: a partir del diagnóstico realizado en la etapa anterior, se realizó una intervención educativa audiovisual al grupo en estudio $(n=80)$. Fue utilizado un video preparado por los autores como instrumento de información y motivación, de los estudiantes; en éste, los estudiantes observaban por un periodo de 60 minutos, la forma correcta de cumplir las metas internacionales de seguridad de pacientes, seguido de ejemplos en que no existía cultura de seguridad en una institución de salud (no reporte frente a un evento adverso, nulo trabajo en equipo, castigo al personal que fue parte de un evento adverso, entre otros).

c) Tercera etapa: se aplicó la encuesta al mes poseducación con el fin de evaluar el efecto educativo mediante el aumento de conocimiento sobre cultura de seguridad de los educandos (variable dependiente); los datos a posteriori fueron analizados estableciendo relaciones entre los datos sociodemográficos de los estudiantes y su desempeño en la encuesta aplicada.

Se realizó análisis estadístico descriptivo, mediante la utilización de medidas de tendencia central, calculando varianza, desviación estándar, rango, curtosis, coeficiente de asimetría, utilizando estadística no paramétrica y estadística paramétrica, contraste de normalidad, prueba de Kolmogorov-Smirnov y de Shapiro-wilk, gráfico q-q normal. Prueba $t$ de Student para muestras relacionadas o pareadas y el contraste de Wilcoxon a través del programa SPSS.

Fueron considerados los siete requisitos éticos de Ezequiel Emanuel descritos para 
este tipo de investigación, cumpliendo los cuatro principios éticos de la investigación en humanos, contando con la autorización del Comité de Ética de la institución universitaria.

\section{RESULTADOS}

Fueron realizadas el 100\% de las actividades, el porcentaje de respuesta de la encuesta correspondió a 82,4\% (80/95 estudiantes). Los datos epidemiológicos de los estudiantes eva- luados fueron: edad promedio 21 años (DT 1.3) (Mínima y máxima de 19 y 25 años), asimetría positiva (valor 1,768 ) y un coeficiente de curtosis de 4,159. En relación a la variable género, el $9 \%$ masculino y un $91 \%$ femenino. El 95\% de alumnos cursaban primera práctica intrahospitalaria y un 5\% extrahospitalaria.

Los resultados de la Tabla 1 muestran que en la sección B, C y E existió un aumento de los conocimientos, evidenciado por una diferencia significativa en los resultados correctos obtenidos por los estudiantes cuando se compara los periodos pre y posintervención.

Tabla 1. Distribución de resultados expresados en medianas de acuerdo al número de respuestas esperadas y obtenidas para los periodos pre y posintervención ( $\mathrm{n}=80$ estudiantes evaluados).

\begin{tabular}{|c|c|c|c|c|c|}
\hline $\begin{array}{l}\text { Sección } \\
\text { evaluada }\end{array}$ & $\begin{array}{l}\text { Descripción } \\
\text { de sección }\end{array}$ & $\begin{array}{c}\text { Puntaje } \\
\text { esperado }^{\star}\end{array}$ & $\begin{array}{l}\text { Preintervención } \\
\text { (Mediana) }\end{array}$ & $\begin{array}{c}\text { Posintervención } \\
\text { (Mediana) }\end{array}$ & p valor \\
\hline B & $\begin{array}{l}\text { Evaluación de los } \\
\text { conocimientos del } \\
\text { estudiante respecto a } \\
\text { seguridad de pacien- } \\
\text { tes }\end{array}$ & 7 & 5,83 & 6,84 & $\mathrm{p}<0,05$ \\
\hline $\mathrm{C}$ & $\begin{array}{l}\text { Evaluación de la per- } \\
\text { cepción sobre el cam- } \\
\text { po clínico en cuanto a } \\
\text { cultura de seguridad }\end{array}$ & 13 & 2,08 & 3,21 & $\mathrm{p}<0,05$ \\
\hline $\mathrm{D}$ & $\begin{array}{l}\text { Valoración de la cul- } \\
\text { tura de seguridad de } \\
\text { los estudiantes }\end{array}$ & 11 & 2,71 & 2,71 & $\mathrm{p}>0,05$ \\
\hline $\mathrm{E}$ & $\begin{array}{l}\text { Valoración de las he- } \\
\text { rramientas y conteni- } \\
\text { dos entregados por la } \\
\text { universidad respecto } \\
\text { a seguridad de pa- } \\
\text { cientes }\end{array}$ & 9 & 2,48 & 2,54 & $\mathrm{p}=0,024$ \\
\hline
\end{tabular}

* Puntaje esperado: puntaje máximo asignado a cada sección. 
Lo que se puede evaluar claramente en la Tabla 2, en la cual se presenta la distribución porcentual promedio de respuestas correctas de ambos periodos evaluados, donde, en cada sección se observa un aumento en puntos porcentuales al comparar el periodo pre y posintervención: Sección $B$, aumentó 46 puntos porcentuales cuando se analizó la "evaluación de los conocimientos del estudiante respecto a seguridad del paciente"; para la sección C, el aumento fue igual a $18 \%$ frente a los ítems dirigidos a analizar la "evaluación de la percepción sobre el campo clínico en cuanto a cultura de seguridad"; finalmente la sección E, "valoración de las herramientas y contenidos entregados por la universidad respecto a seguridad de pacientes", aumentó 20 puntos porcentuales en la opinión de los estudiantes posintervención.

Tabla 2. Distribución porcentual en promedio de respuestas correctas, respecto a la pre y posintervención ( $\mathrm{n}=80$ estudiantes).

\begin{tabular}{ccc}
\hline \multirow{2}{*}{ Secciones } & \multicolumn{2}{c}{ Respuestas correctas (\%) } \\
& Preintervención & Posintervención \\
\hline B & 44 & $90^{\star}$ \\
C & 13 & $31^{\star}$ \\
D & 51 & 54 \\
E & 14 & $34^{\star}$ \\
\hline Promedio & 30,5 & 52,3 \\
\hline
\end{tabular}

${ }^{\star}$ Diferencia entre los periodos pre y posintervención con valores de $\mathrm{p}<0,005$

Los datos del Gráfico 1 muestran los valores medios pre y posintervención en relación a la evaluación de la sección B "conocimientos del estudiante respecto a seguridad de pacientes", concluyendo que, a través de las pruebas de Rangos con Signos de Wilcoxon, las mediciones de evaluación de los conocimientos del estudiante respecto a seguridad de pacientes pre y posintervención presentan diferencias significativas $(\mathrm{p}<0,05)$.

Los datos del Gráfico 2 muestran los valores medio pre y posintervención en la evaluación de la percepción sobre el campo clínico en cuanto a cultura de seguridad (Sección C), a través de la prueba $\mathrm{T}$ para muestras relacionadas, las mediciones de evaluación pre y posintervención presentan diferencias significativas $(p<0,025)$. Esto nos permite definir que el video instruccional fue efectivo, aumentando en promedio 1,13 puntos la percepción de los estudiantes sobre el campo clínico en cuanto a cultura de seguridad.

Los datos del Gráfico 3 evidencian los valores de tendencia central pre y posintervención, no presentan diferencia entre las respuestas correctas, especialmente en el valor de la mediana de los puntajes. Al aplicar la prueba de Shapiro Wilk, para muestras pequeñas, se presenta un nivel de significancia igual a 0,055 para la valorización de la cultura de seguridad de los estudiantes en el pre y posintervención respectivamente. En consecuencia no existe impacto del video educativo.

El gráfico 4 muestra un diagrama de cajas, pre y posintervención, de la sección E "valoración sobre las herramientas y contenidos entregados por la universidad respecto a seguridad de pacientes", evidenciando que los valores de tendencia central pre y posintervención, presentan una diferencia muy pequeña entre las respuestas obtenidas, es- 
pecialmente en el valor de los puntajes medios. A través de la aplicación de la Prueba de Wilcoxon de muestras relacionadas, las mediciones pre y posintervención presentan diferencias significativas $(\mathrm{p}=0,024)$, para la valoración sobre las herramientas y contenidos entregados por la universidad respecto a seguridad de pacientes.

Gráfico 1. Valores medios pre y posintervención frente a la evaluación de la sección B "Evaluación de los conocimientos del estudiante respecto a la seguridad del paciente" (muestra para periodos pre y posintervención $=80$ estudiantes).

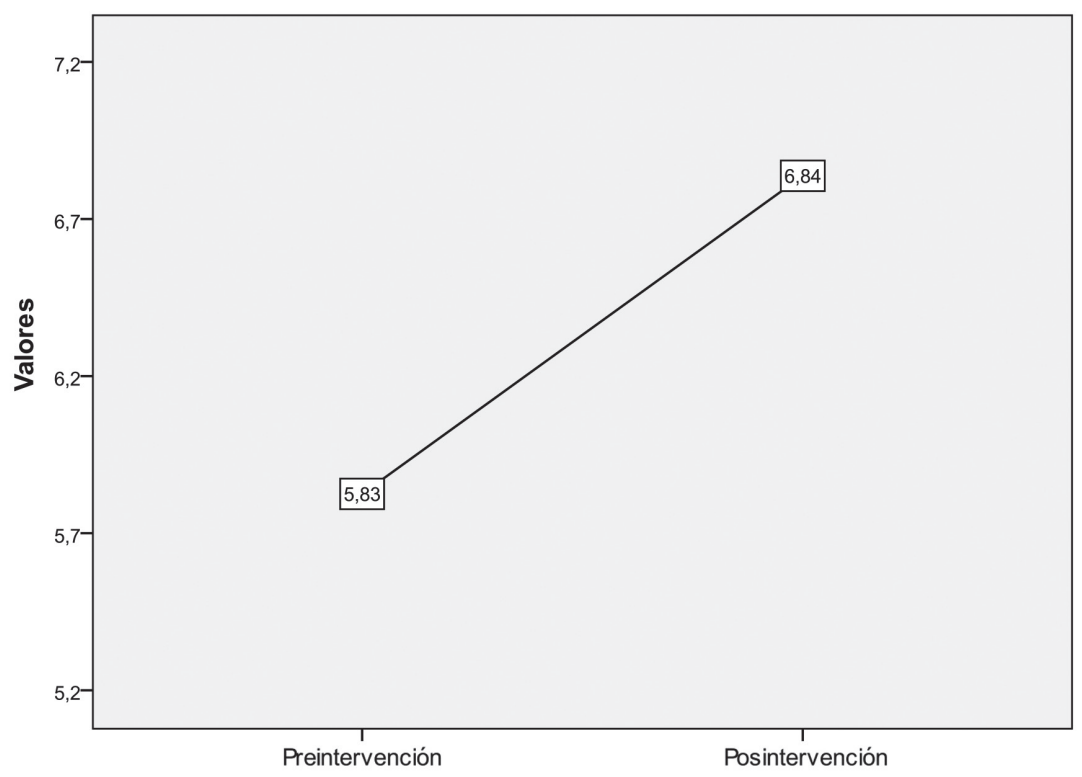

Gráfico 2. Diagrama de cajas pre y posintervención sección C "Evaluación de la percepción sobre el campo clínico en cuanto a cultura de seguridad" (muestra para periodos pre y posintervención $=80$ estudiantes).

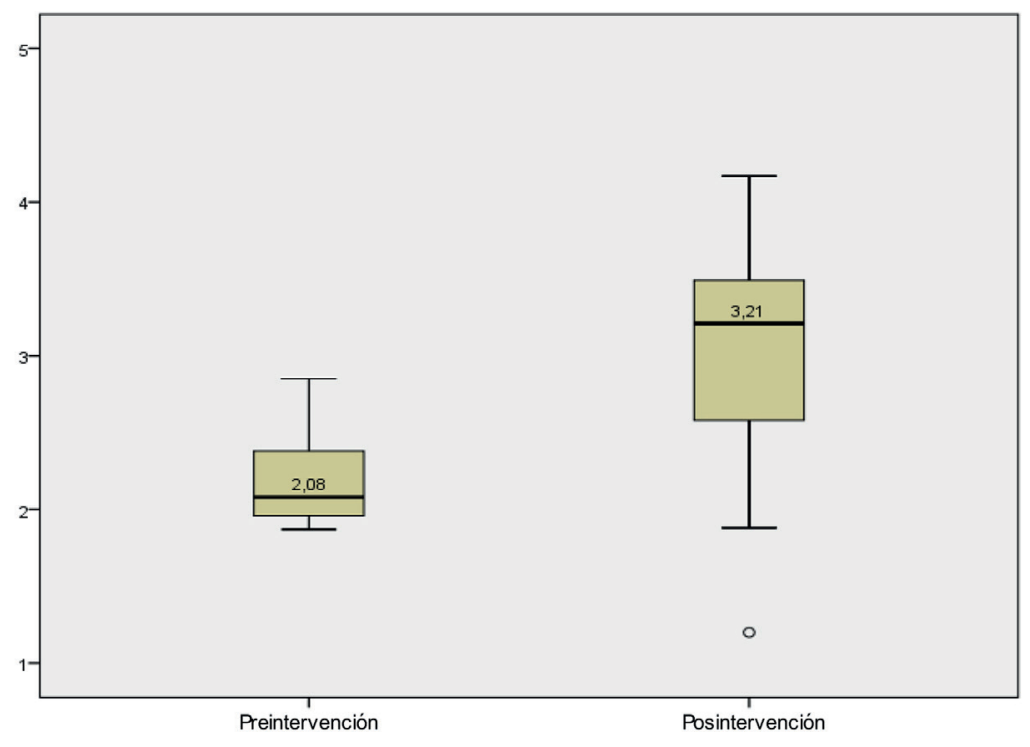


Gráfico 3. Diagrama de cajas pre y posintervención sección D "Valoración de la cultura de seguridad de los estudiantes" (muestra para periodos pre y posintervención $=80$ estudiantes).

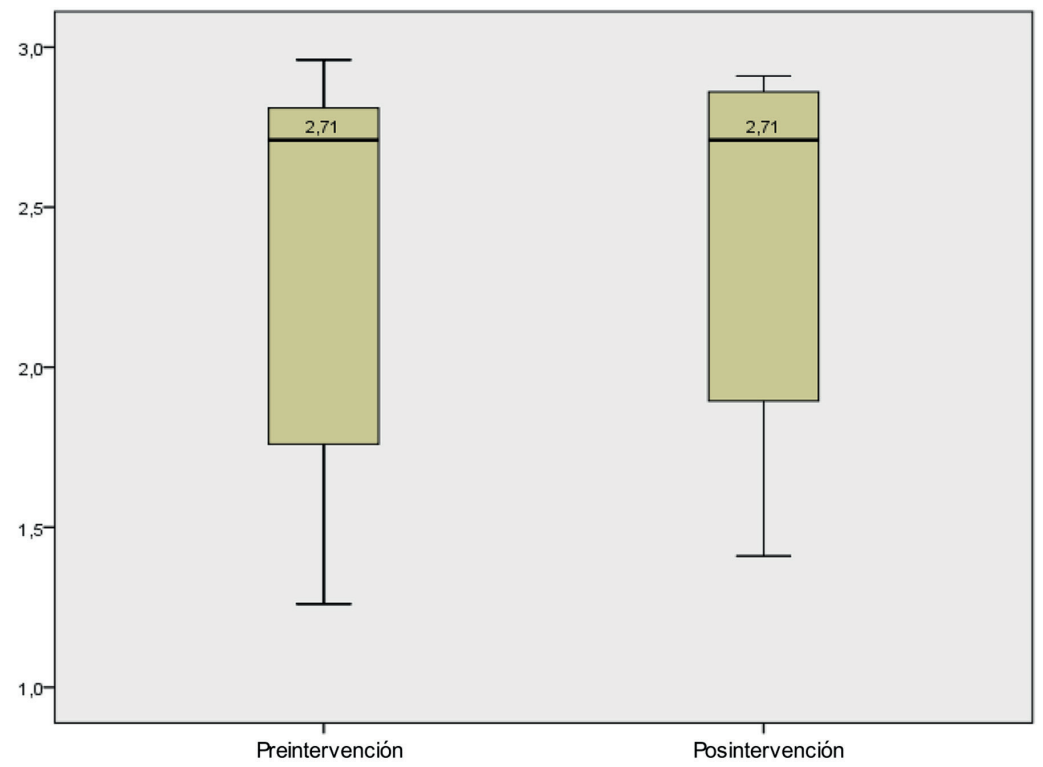

Gráfico 4. Diagrama de cajas pre y posintervención sección E "Valoración de las herramientas y contenidos entregados por la universidad respecto a seguridad de pacientes" (muestra para periodos pre y posintervención $=80$ estudiantes).

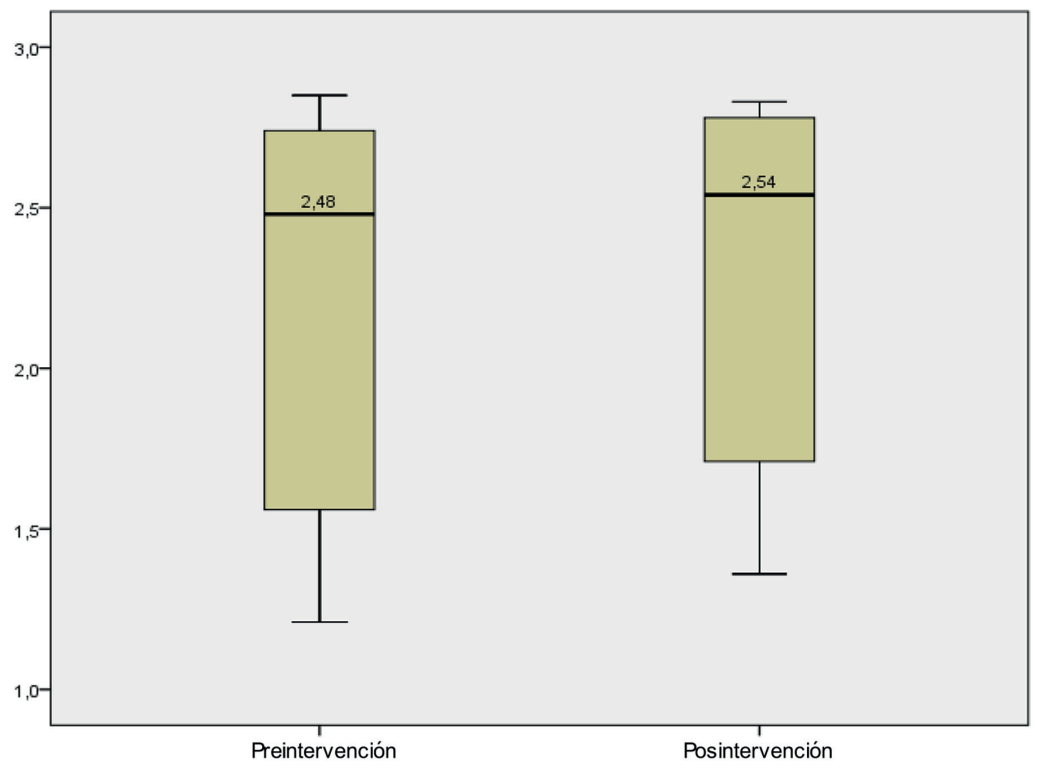




\section{DISCUSIÓN Y CONCLUSIÓN}

El presente estudio evaluó, mediante la aplicación de una encuesta elaborada por los autores, una intervención educativa audiovisual, estructurada sobre temas relevantes de seguridad del paciente (metas internacionales de seguridad de pacientes y cultura de seguridad) dirigida a estudiantes de Enfermería de pregrado, en su primera práctica clínica. Aquí se discute la utilidad del instrumento creado, para realizar la evaluación antes y después de la intervención, el efecto del video instruccional, elaborado a partir de las respuestas de los estudiantes. Finalmente se analizarán las variaciones en las respuestas de las secciones evaluadas en los periodos pre y posintervención.

En el presente trabajo se observa que el video instruccional creado permitió aumentar las respuestas correctas de los estudiantes para los periodos pre y posintervención en 30,5 a $52,3 \%$ respectivamente $(\mathrm{p}<0,05)(\mathrm{Ta}-$ bla 2). Este efecto global puede ser comparado con el estudio realizado el año 2014, donde se comprobó que las tecnologías educativas (videos) mejoran la formación de profesionales de salud en cuanto a los resultados de aprendizaje del estudiante, logrando alcanzar cognitiva y afectivamente los objetivos propuestos desde el punto de vista del educando con y sin video $(4,2$ y $3,3 \%$, respectivamente, $\mathrm{p}<0,001)(13)$.

Otros autores consideran que el video es un medio audiovisual que permitió mostrar a los estudiantes un conjunto de técnicas visuales y auditivas que apoyaron la enseñanza, facilitando una mayor y más rápida comprensión e interpretación de las ideas (14).

Se destaca que el porcentaje de respuesta de la encuesta correspondió a 82,4\% (80/95 estudiantes), esto implica que los estudiantes que participaron del presente estudio tienen interés en el tópico de seguridad; al evaluar otros resultados de aplicación de encuestas en el mismo tema, se observan porcentajes de respuesta entre 80 y $92 \%(17,18)$.

Prevenir los eventos adversos y mejorar la seguridad del paciente en la atención de la salud son objetivos clave de la educación de enfermería (7). La calidad, el contenido y la oferta de la educación de Enfermería pueden tener un impacto significativo en los comportamientos de seguridad de los futuros estudiantes en sus entornos clínicos.

En estos últimos años se han desarrollado instrumentos orientados a establecer la conciencia, las habilidades y las actitudes percibidas de los estudiantes de Enfermería de pregrado, en cuanto a la educación sobre la seguridad del paciente que entregan las Escuelas de enfermería: 1) El instrumento Healthcare Professionals Patient Safety Assessment Curriculum Survey (HPPSACS), aplicado el año 2015 a estudiantes en formación de Enfermería, presentó un alfa de Cronbach de 0,64 , lo que indica una consistencia interna moderada del instrumento (19); 2) La versión italiana de la "Encuesta de Educación Profesional de la Salud en la Seguridad del Paciente" (H-PEPSSIta), cuyos autores concluyen que es una herramienta válida que evalúa la autopercepción de los estudiantes de Enfermería, en relación con el conocimiento y la competencia de la seguridad del paciente (18); 3) Encuesta de evaluación de la calidad de la educación para Enfermería (QSEN), cuyos resultados indicaron que la educación para la seguridad del paciente fue impartida como actividades extracurriculares (17).

Las encuestas anteriores se orientan a establecer cómo se entregan los conocimientos por parte de las escuelas de Enfermería, en el tema de seguridad clínica, sin embargo, la presente investigación evalúo una nueva encuesta, destinada a determinar las necesidades de conocimientos de los estudiantes, que realizan su primera práctica clínica. Y en base a éstas, estructurar un video educativo en temas básicos de seguridad (metas internacionales de seguridad del paciente y cultura de seguridad), destacando que el alfa de 
Cronbach fue igual a 0,82 , lo que indica una consistencia interna buena del instrumento creado.

En el año 2011 la OMS lideró las actividades tendientes a potenciar la educación en seguridad de pacientes para la formación de la futura generación de trabajadores de la salud, con un solo propósito, ejercer una atención clínica centrada en el paciente, estructurando cinco objetivos específicos: 1) Preparar a los estudiantes de carreras de la salud para una práctica segura; 2) Informar a las facultades de salud sobre los temas principales en materia de seguridad; 3 ) Potenciar la seguridad del paciente como tema de todas las carreras de salud; 4) Brindar un programa integral tendiente a asistir en la enseñanza y la integración del aprendizaje en seguridad; 5) Fomentar el desarrollo de capacitación en seguridad del paciente (9).

La encuesta aplicada en este trabajo presentaba cinco secciones, para la presente discusión no se tomará en cuenta la Sección A, que corresponde a datos epidemiológicos de los estudiantes. En relación a los resultados obtenidos, en base a la intervención educativa audiovisual aplicada al grupo en estudio, se observa que tres de las cuatro secciones de la encuesta mostraron un efecto educativo en el conocimiento de los estudiantes pre y posintervención en las siguientes secciones: Sección B "evaluación de los conocimientos del estudiante respecto a seguridad de pacientes"; Sección C "evaluación de la percepción sobre el campo clínico en cuanto a cultura de seguridad" y Sección E "valoración sobre las herramientas y contenidos entregados por la universidad respecto a seguridad de pacientes”. No así en la Sección D, orientada a evaluar la "valoración de la cultura de seguridad de los estudiantes".

En el año 2016 se realizó un estudio transversal que evalúa estudiantes de Enfermería, para determinar cómo y en qué medida la educación sobre el tema seguridad del paciente fue entregada. Los autores concluyeron que existe la necesidad de revisar el cu- rrículo de enfermería y utilizar diversos métodos de enseñanza para impartir educación sobre la seguridad del paciente más completa y eficazmente, destacando que es necesario desarrollar un enfoque integrado para garantizar la competencia equilibrada en los estudiantes (17).

Evaluar críticamente el contenido de la seguridad del paciente en la educación de estudiantes de Enfermería fue el objetivo de una revisión integrativa de la literatura, la que reveló, los contenidos identificados como importantes fueron: eventos adversos, la acción anticipatoria en entornos complejos y la enfermería centrada en la seguridad del paciente, la sensibilidad al papel de los estudiantes de enfermería (7).

La seguridad del paciente debe ser entendida como una responsabilidad esencial del acto del cuidado, de ahí que los profesionales de enfermería debamos sumarnos a las nuevas estrategias que garanticen un cuidado de calidad y seguro (20); los cuidados enfermeros es una de las prestaciones donde se establece contacto directo con el usuario. En ese entendido, el diseño de un plan de enfermería supone tener en cuenta el riesgo específico de cada paciente, así como el contexto organizacional en el cual se actúa profesionalmente (12).

En conclusión, los resultados obtenidos en base a la intervención educativa audiovisual al grupo en estudio (estudiantes de Enfermería en su primera práctica clínica) muestran que existió un aumento de conocimiento de los educandos en temas básicos de seguridad clínica. De esta manera, de las 5 secciones de la encuesta, se tiene una efectividad en los temas metas internacionales de seguridad de pacientes (sección B); percepción sobre el campo clínico en cuanto a cultura de seguridad (sección C) y valoración sobre las herramientas y contenidos entregados por la universidad respecto a seguridad de pacientes (sección E).

Los docentes de enfermería debemos comprender la importancia de este tema a 
nivel mundial y producir un cambio cultural en pregrado; además se requiere desarrollar la investigación en este tema, en especial medir el conocimiento y percepción de los estudiantes en el tópico de seguridad, y determinar cuáles son las mejores técnicas educativas para que los estudiantes de pregrado adquieran los conocimientos y competencias necesarios en seguridad del paciente.

\section{Limitaciones del estudio}

Si bien en las distintas secciones hubo resultados satisfactorios, es importante mencionar que estas diferencias significativas se encontraron al borde del límite de significancia, en especial la sección E, por lo que los datos se deben interpretar con cautela. Una segunda limitación es que no existió evaluación en relación a la aplicación de los conocimientos a posteriori de los estudiantes que fueron educados con el video, lo cual impide evaluar el impacto en el cambio de conducta de los estudiantes a los tres y seis meses de la educación.

\section{REFERENCIAS}

1. Organización Mundial de la Salud. La investigación en seguridad del paciente, Mayor conocimiento para una atención más segura [monografía en internet]. Ginebra: OMS; 2008 [citada 10 jul 2013]. Disponible en: http://www.who.int/patientsafety/information_centre/documents/ps_research_brochure_es.pdf

2. Ministerio de Salud de Chile. Ley No 19.937/2004 de 30 de enero. Modifica el D.L. No 2.763 , de 1979, con la finalidad de establecer una nueva concepción de la autoridad sanitaria, distintas modalidades de gestión y fortalecer la participación ciudadana [citada 10 de jul 2013]. Disponible en: https://www.leychile.cl/ Navegar?idNorma $=221629$ \&idParte $=0$
3. Superintendencia de Salud. Informa avances instrumentos de evaluación de calidad en salud. Oficio circular IP $/ \mathrm{N}^{\circ} 1$, 30 diciembre 2008.

4. Aranaz-Andrés JM, Aibar-Remon C, Limón-Ramírez R, Amarilla A, Restrepo FR, Urroz O, et al. Prevalence of adverse events in the hospitals of five Latin America countries: results of the "Iberoamerican study of adverse events" (IBEAS). BMJ Qual Saf. 2011; 20(12): 1043-51.

5. León-Román C. La seguridad del paciente, una responsabilidad en el acto del cuidado. Rev Cubana Enferm [Internet]. 2006 [citada 10 de abr 2017]; 22 (3). Disponible en: http://bvs.sld.cu/revistas/enf/ vol22_3_06/enf07306.html

6. Bianchi M, Bressan V, Cadorin L, Pagnucci N, Tolotti A, Valcarenghi D, et al. Patient safety competencies in undergraduate nursing students: a rapid evidence assessment. J Adv Nurs. 2016; 72(12): 2966-2997.

7. Tella S, Liukka M, Jamookeeah D, Smith NJ, Partanen P, Turunen $H$. What do nursing students learn about patient safety? an integrative literature review. J Nurs Educ. 2014; 53(1): 7-13.

8. Gregory A, Debourgh E, Anef Y, Susan K, Prion E. Patient safety manifesto: a professional imperative for prelicensure nursing education. J Prof Nurs. 2012; 28(2): 110-118.

9. World Health Organization. WHO patient safety curriculum guide: multi-professional edition. WHO Library Cataloguing-in-Publication [Internet]. Ginebra: WHO; 2011 [citada 6 oct 2013]. Disponible en: http://apps.who.int/iris/bitst ream/10665/44641/1/9789241501958 eng.pdf

10. Organización Mundial de la Salud (OMS). Marco conceptual de la clasificación Internacional para la seguridad del Paciente: [monografía en internet] Ginebra: OMS; 2009 [citada 10 jul 2013]. Disponible en: http://www.who.int/pa- 
tientsafety/implementation/icps/icps_ full_report_es.pdf

11. Organización Mundial de la Salud. Nueve soluciones para la seguridad del paciente [Internet]. Washington/Ginebra: OMS; 2007 [citada 5 jul 2013]. Disponible en: http://www.who.int/mediacentre/news/ releases/2007/pr22/es/

12. Gómez A, Espinosa A. Dilemas éticos frente a la seguridad del paciente: cuidar es pensar. Rev Aquichán. 2006; 6(1): 5467.

13. Zarza-Arizmendi MD, Alba-Leonel A, Arturo Salcedo-Álvarez R. El currículum de enfermería y la seguridad del paciente. Rev CONAMED. 2008; 13: 33-37.

14. Cooper E. Nursing student medication errors: a snapshot view from a school of nursing's quality and safety officer. J Nurs Educ, 2014; 53(3): s51-4.

15. Organización Mundial de la salud/ The Joint Commission. Preámbulo a las soluciones para la seguridad del paciente [Internet]. Ginebra: OMS; 2007 [citada 6 jun 2013]. Disponible en: http://www.jointcommissioninternational.org/assets/3/7/ PatientSolutionsSpanish.pdf
16. Joint Commission International. Estándares de acreditación de los hospitales de Joint Commission International. $5^{\text {a }}$ ed. Illinois: Joint Commission International; 2014. $326 \mathrm{p}$.

17. Lee NJ, Jang H, Park SY. Patient safety education and baccalaureate nursing students' patient safety competency: A cross-sectional study. Nurs Health Sci. 2016; 18(2): 163-71.

18. Stevanin S, Bressan V, Bulfone G, Zanini A, Dante A, Palese A. Knowledge and competence with patient safety as perceived by nursing students: The findings of a cross-sectional study. Nurse Educ Today. 2015; 35(8): 926-34.

19. Mansour M. Factor analysis of nursing students' perception of patient safety education. Nurse Educ Today. 2015; 35(1): 32-7.

20. Ferreira-Umpiérrez A, Fort-Fort Z, Chiminelli-Tomás V. Eventos adversos en salud y cuidados de enfermería: la seguridad del paciente desde la experiencia del profesional. Texto contexto-enferm. 2015; 24(2): 310-5. 